\title{
A New Neural Network Technique for the Design of Multilayered Microwave Shielded Bandpass Filters
}

\author{
Juan Pascual García, Fernando Quesada Pereira, David Cañete Rebenaque, \\ Juan Sebastián Gómez Díaz, Alejandro Álvarez Melcón
}

Department of Information Technologies and Communications (TIC), Technical University of Cartagena, Cartagena, Murcia, Antiguo Cuartel de Antiguones 30202, Spain

Received 16 May 2008; accepted 27 October 2008

\begin{abstract}
In this work, we propose a novel technique based on neural networks, for the design of microwave filters in shielded printed technology. The technique uses radial basis function neural networks to represent the non linear relations between the quality factors and coupling coefficients, with the geometrical dimensions of the resonators. The radial basis function neural networks are employed for the first time in the design task of shielded printed filters, and permit a fast and precise operation with only a limited set of training data. Thanks to a new cascade configuration, a set of two neural networks provide the dimensions of the complete filter in a fast and accurate way. To improve the calculation of the geometrical dimensions, the neural networks can take as inputs both electrical parameters and physical dimensions computed by other neural networks. The neural network technique is combined with gradient based optimization methods to further improve the response of the filters. Results are presented to demonstrate the usefulness of the proposed technique for the design of practical microwave printed coupled line and hairpin filters. (C) 2008 Wiley Periodicals, Inc. Int J RF and Microwave CAE 19: 405-415, 2009.
\end{abstract}

Keywords: neural networks; filter design; multilayered shielded structures; microwave filters

\section{INTRODUCTION}

Neural networks constitute one useful technique in microwave filter modeling and design. The first applications of neural networks were focused on modeling different microwave devices. In the design task, neural networks can be employed following two different strategies. The first approach consists on using the neural networks employed in the modeling task in an inverse way [1]. In the second method, neural inputs and outputs are interchanged with respect to the modeling methodology. Thus, the inputs correspond to the electrical response, and the outputs are the physical

Correspondence to: J. P. García; e-mail: juan.pascual@upct.es DOI 10.1002/mmce.20363

Published online 23 December 2008 in Wiley InterScience (www.interscience.wiley.com). dimensions. Therefore, this strategy is known as direct inverse modeling. Some works have proved the capability of neural networks to design microwave devices such as, i.e., horn antennas [2] and filters [3]. In Refs. 4, 5, neural networks are used to approximate the relations between some elements of a filter coupling matrix and the physical dimensions that synthesize the mentioned coupling elements. The technique was applied to design several waveguide pseudoelliptic filters. Neural networks have also been applied to printed multilayer filter design. In Ref. 6, the traditional multilayer perceptron neural network is utilized to obtain the geometrical dimensions of coupled line filters, using as inputs some normal mode parameters (such as impedance and voltage ratios).

Two of the most popular transfer functions used in the design of practical filters are the Butterworth and the Chebyshev responses. The lowpass prototype 
filter elements corresponding to a given desired response permit to evaluate the external quality factors and the coupling coefficients between all the resonators of the filter [7]. The main difficulty in the final design of the filter lies on finding the resonators dimensions, which synthesize the computed quality factor and coupling coefficients. In the case of microstrip filters printed on substrates, with infinite transverse dimensions, there exist empirical formulas that allow to calculate the resonator dimensions using look-up tables. For example, in the case of parallel coupled lines, the dimensions are computed from the even and odd impedance values [8]. In other cases, the physical dimensions of the filter are obtained from design curves that relate these dimensions with the quality factors, coupling coefficients, and other electrical parameters [8-10].

In shielded multilayered microstrip filters, the transverse dimensions are finite, and there are no empirical equations to compute easily the physical dimensions of the filter. If the empirical equations corresponding to the unshielded structure are employed, the required precision is not usually attained. This is because the shielding effects and the interactions with the lateral walls are neglected. The construction of new design curves for shielded printed filters requires the calculation of a high number of quality factors and coupling coefficient values, to reach enough precision in the filter design task. These calculations can be performed using full-wave analysis techniques, such as the integral equation or the finite elements method. However, such full-wave analysis techniques usually consume high computational resources.

In this work, we propose the use of radial basis function neural networks to learn the relations (in a given frequency bandwidth) between the quality factor, resonant frequency, and coupling coefficients, with their corresponding physical dimensions. In this way, a limited number of quality factors and coupling coefficients are needed to generate the information required for the design of practical filters. Using the novel technique, an initial amount of time is needed to generate the neural networks training and testing data. Furthermore, to largely reduce the training time, the neural network method developed in Ref. 11 is used during the calculation of the training data. Once trained, different printed multilayer filters with center frequency inside the operating bandwidth can be easily designed. The output of the neural network will be, directly, the dimensions of the filter which synthesize a given transfer function. It is important to remark that the training set generation and neural training times are not included in the filter design step. When a new filter is designed using the neural networks, it is necessary neither to generate a new training set nor to train the neural networks.

If the designed filter does not fulfill totally the specifications, then a fast optimization step is performed based on gradient techniques. These methods have been widely applied to the design of microwave circuits, i.e., in microstrip structures as in Refs. 12, 13. Generally, the last step in a design process is the optimization of an initial geometry [14]. The neural method described in this article provides an initial geometry very close to the optimum. This will drastically reduce the time invested in the optimization, assuring at the same time a proper convergence of gradient base algorithms (which strongly relies on a very good initial point). The present method is not limited to a specific type of printed filter as in Ref. 6. As results will show, different hairpin and coupled line filters can be designed employing the same methodology.

\section{THEORY}

Different steps are needed for the design of microwave filters. First, the order of the filter is computed from the specifications and from the desired transfer function characteristics (typical transfer functions include Butterworth, Chebyshev or Elliptic functions). Second, the quality factors and coupling coefficients are calculated with the element values of the synthesized lowpass prototype filter. Finally, the dimensions of the resonators are calculated to synthesize the required external quality factors and coupling coefficients [7].

This last step is the most important and complicated to carry out. Depending on the type of resonator chosen to implement the filter, different structures can be employed to calculate the quality factors and coupling coefficients. Doubly and singly terminated resonator structures can be used for the evaluation of the external quality factor [8]. A doubly loaded configuration can be employed in coupled line resonators, as seen in Figure 1a.

This structure posses a transmission $\left(S_{21}\right)$ parameter response (see Fig. 2), which permits the computation of the resonant frequency and $3 \mathrm{~dB}$ bandwidth. Using these parameters the quality factor is calculated as follows [8]:

$$
Q_{\mathrm{e}}=\frac{2 f_{\mathrm{o}}}{\Delta f_{3 \mathrm{~d} \mathrm{~B}}}
$$

For the characterization of hairpin line resonators, instead, it is more convenient to use a singly loaded structure, as illustrated in Figure 3a. 


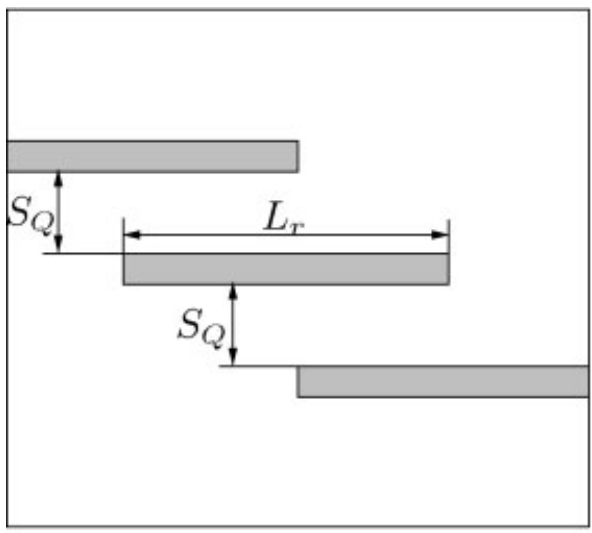

a)

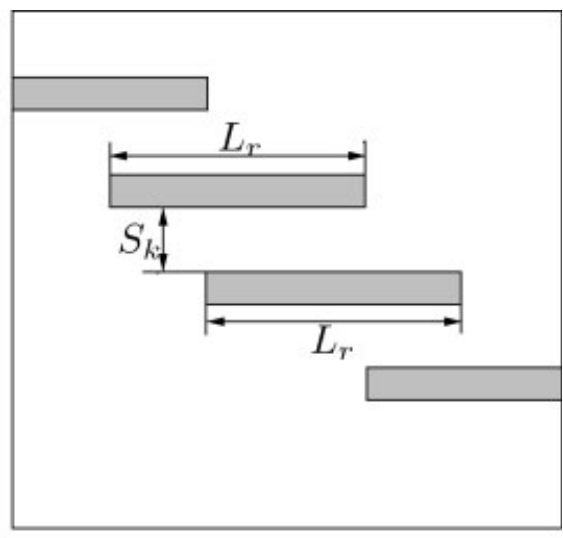

b)

Figure 1. Microstrip structures employed to calculate the quality factor (a) and coupling coefficients (b) when coupled line resonators are chosen.

The reflection coefficient $\left(S_{11}\right)$ phase response is employed to obtain the resonant frequency. The external quality factor is determined by those frequencies at which the phase is $\pm 90^{\circ}$ with respect to the phase at the resonant frequency. The resonant frequency can be computed from the derivative of the reflection coefficient phase, as seen in Figure 4. When these parameters are properly calculated the quality factor is computed as follows [8]:

$$
Q_{\mathrm{e}}=\frac{f_{\mathrm{o}}}{\Delta f_{ \pm 90^{\circ}}}
$$

In addition, the coupling coefficient is computed employing the two coupled resonators structure of Figures $1 \mathrm{~b}$ and $3 \mathrm{~b}$. Two frequencies, $f_{\mathrm{p} 1}$ and $f_{\mathrm{p} 2}$, are extracted from the transmission coefficient magnitude $\left(S_{21}\right)$ response, as shown in Figure 5. These resonant frequencies are used in the evaluation of the coupling coefficient, using [8]:

$$
k=\frac{f_{\mathrm{p} 2}^{2}-f_{\mathrm{p} 1}^{2}}{f_{\mathrm{p} 2}^{2}+f_{\mathrm{p} 1}^{2}}
$$

Although coupled and hairpin line resonators have been used to show how to compute the quality factors and coupling coefficients, any other kind of resonators can also be employed, leading to the same conclusions [8]. In this work, synchronously tuned resonators with Chebyshev characteristics are exploited. More elaborated asynchronously tuned transfer functions can also be considered, with similar level of complexity.

As it was said in the introduction, design curves, which relate the quality factors and coupling coefficients to the physical dimensions, can be generated to help during the design task. To achieve a significant precision, it is necessary to calculate an elevated number of points. Neural networks are able to capture the nonlinear relations in multidimensional data sets. Therefore, neural networks are appropriate to substitute the design curves using a reduced number of data. The idea, which we propose, is to use neural networks that take as inputs the electrical parameters (quality factors, resonant frequency, and coupling coefficients) and offer as outputs the resonator physical dimensions. In general, $N$ neural networks with different inputs and outputs will be needed to calculate the filter dimensions, as seen in Figure 6.

In the case of coupled line filters (see Fig. 1), two neural networks are needed $(N=2)$. As seen in

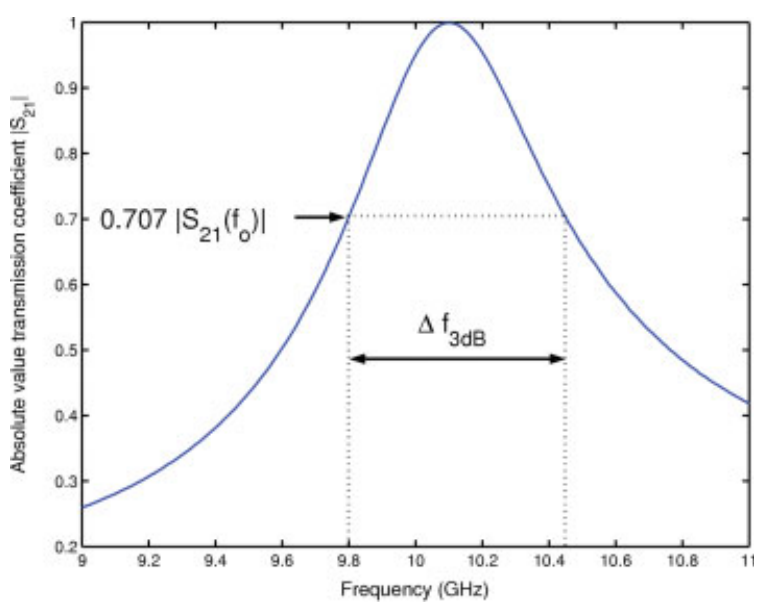

Figure 2. Example of the transmission coefficient $\left(S_{21}\right)$ response employed to compute the external quality factor when a doubly loaded structure is used. [Color figure can be viewed in the online issue, which is available at www.interscience.wiley.com]. 


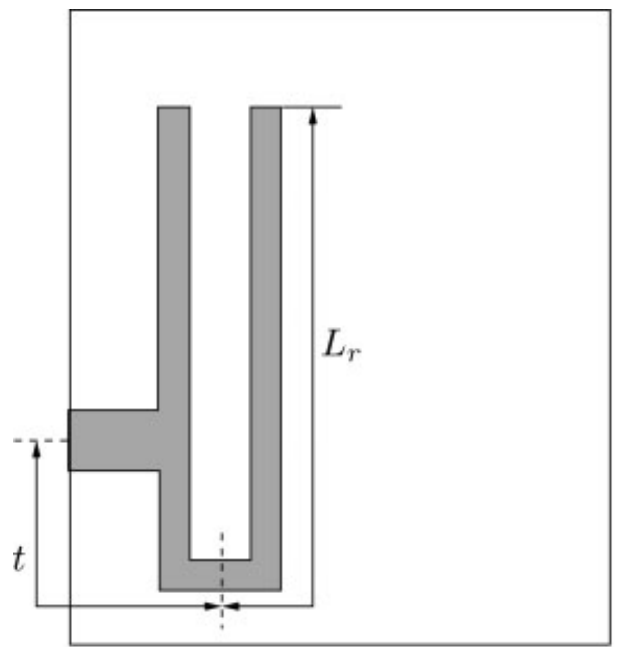

a)

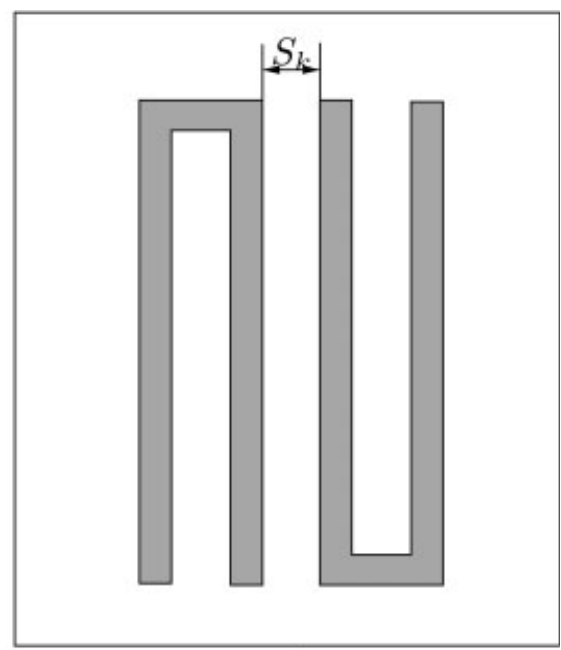

b)

Figure 3. Microstrip structures employed to calculate the quality factor (a) and coupling coefficients (b) when hairpin resonators are chosen.

Figure 7, the first neural network computes the relation between the coupling gap $\left(S_{\mathrm{Q}}\right)$ to the external quality factor.

The neural network also calculates the relation between the length of the resonator $\left(L_{\mathrm{r}}\right)$ to its resonant frequency $f_{\mathrm{c}}$ (see Fig. 7). The second neural network learns the relations between the desired coupling coefficients for different resonator lengths, with the resonator gap separation $\left(S_{\mathrm{K}}\right)$ that synthesizes the given coupling coefficient. As seen in Figure 7, the two neural networks are connected in cascaded configuration. It is important to remark that the second neural network is trained with different sampled reso-

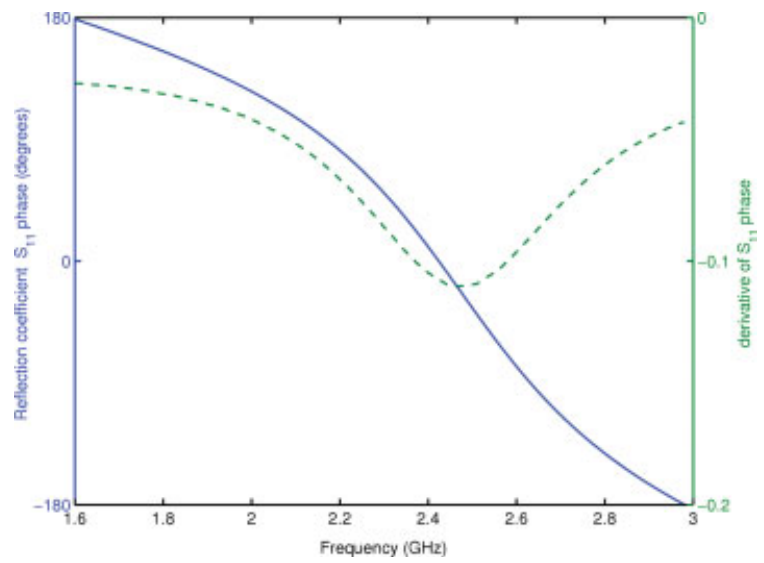

Figure 4. Example of reflection coefficient $\left(S_{11}\right)$ response (continuous line) and its derivative (dash line) employed to compute the external quality factor when a singly loaded structure is used. [Color figure can be viewed in the online issue, which is available at www.interscience.wiley.com]. nator lengths, but when it is employed to compute the gap separation $S_{\mathrm{K}}$, it uses as input the length calculated with the first neural network. This is why in Figure 7 the output of the first neural network is connected to the input of the second neural network. The outputs are directly the dimensions of the different resonators needed to synthesize a given transfer function. Following the model shown in Figure 6, for coupled line resonators, the first neural network has two inputs $(K=2)$ and two outputs $(M=2)$, whereas the second neural network possess two inputs $(P=$ 2) and one output $(Q=1)$.

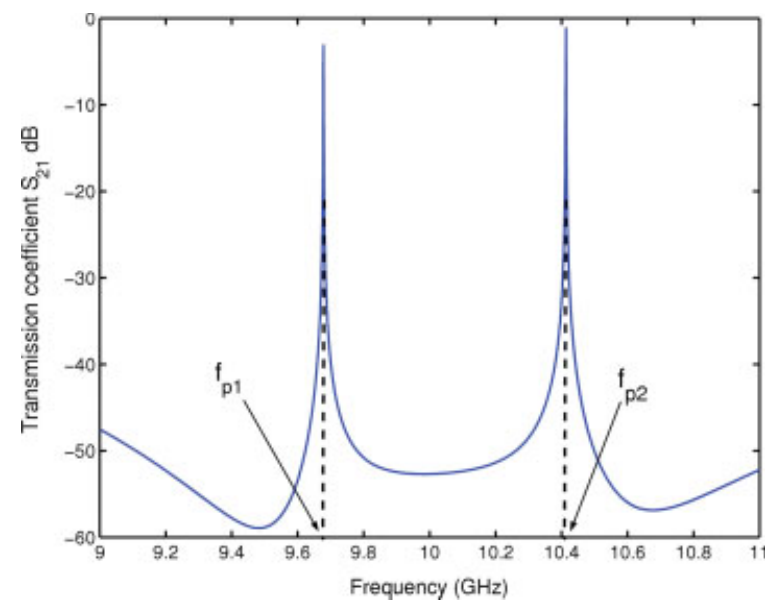

Figure 5. Example of the transmission coefficient $\left(S_{21}\right)$ response corresponding to the structures of Figures 1(b) and 3(b). [Color figure can be viewed in the online issue, which is available at www.interscience.wiley.com]. 


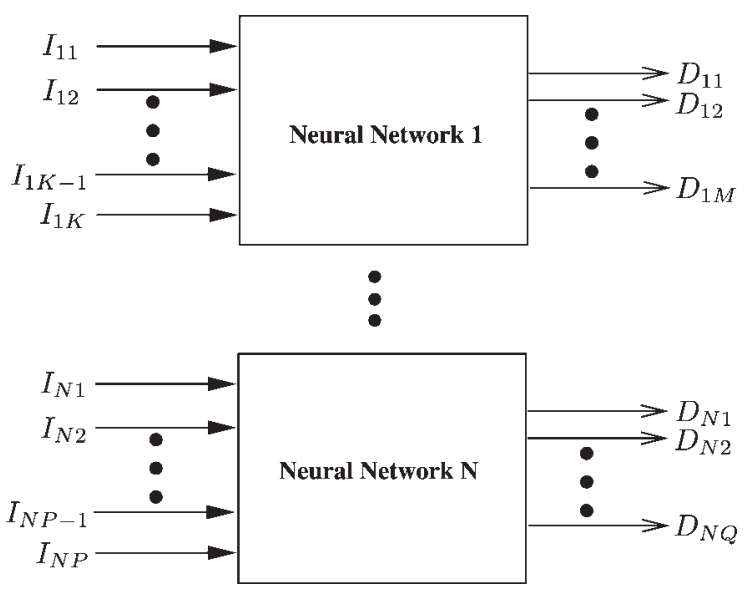

Figure 6. Neural networks general operation in the design task. Up to $N$ neural networks can be needed to calculate the desired physical dimensions. Each neural network takes a particular set of inputs $(I)$ composed of a number of electrical parameters and physical dimensions. Each one of the physical dimensions at the input level can be either preset by the user or calculated by a previous neural network. Each neural network delivers a specific set of physical dimensions $(D)$ different from the outputs of the rest of neural networks. These physical dimensions synthesize the electrical parameters of the corresponding neural network input. One or more of the $D$ outputs of a neural network can be connected to other neural networks as inputs.

Hairpin line filters (see Fig. 3) also employ two neural networks $(N=2)$. The first neural network captures the relations between the tapping length $(t)$ to the external quality factor. Moreover, this neural network allows the calculation of the relation between the resonator length $\left(\mathrm{L}_{\mathrm{r}}\right)$ and the corresponding resonant frequency $f_{\mathrm{c}}$. The second neural network operates in a similar way when compared with the second neural network described for the coupled line resonator case. This neural network computes the relations between the resonator lengths and coupling coefficients with the resonator gap separations $\left(S_{K}\right)$.

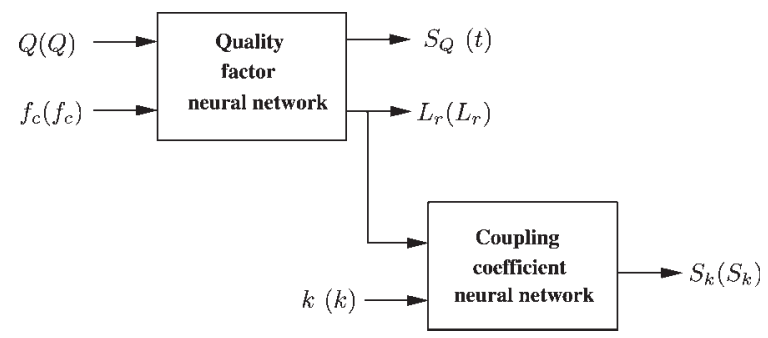

Figure 7. Neural Networks structure when either coupled line resonators or hairpin line resonators (values in brackets) are used.
Both neural networks are connected again in cascade configuration. Therefore, the neural networks used to calculate the hairpin filter dimensions posses a similar structure as the neural networks used for coupled line resonators $(K=2, M=2, P=2$, and $Q=1)$ (see Fig. 7).

The methodology applied to coupled line and hairpin resonators can be employed to any other kind of resonators. The number of neural networks is not limited to two. Depending on the situation, the neural structure can be adapted following the general model sketched in Figure 6. Furthermore, each neural network is allowed to posses a particular set of inputs and outputs. The final neural structure is very flexible, because it can contain cascade configurations as shown in Figure 7.

\section{NEURAL NETWORK PERFORMANCE}

The radial basis function neural network has been selected to show the capabilities of the neural networks to calculate the dimensions of the resonators. Coupled line resonators, as the ones shown in Figure 1 , were selected for the design of a four-pole filter. The multilayered shielded structure is sketched in Figure 8. In addition, hairpin resonators were chosen to design a fifth order filter inside the boxed structure, as shown in Figure 9.

The quality factors and coupling coefficients needed as training and testing sets for both boxes were computed using the neural network method described in Ref. 11. In this method, the Green's functions of the multilayered structure are further approximated by a set of neural networks. If other slow full-wave techniques are used during the generation of the neural training and testing sets, large computational times are required, making more difficult the application of the present method.

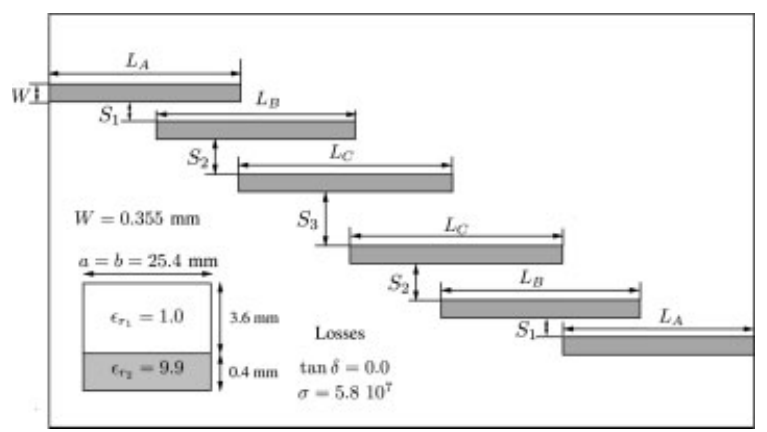

Figure 8. Multilayered shielded structure and fourth order filter. 


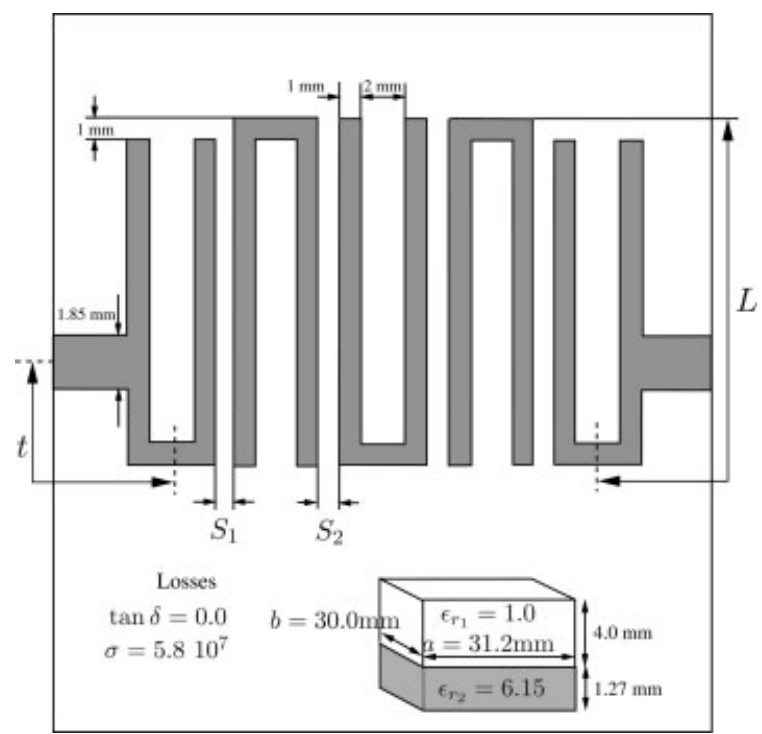

Figure 9. Multilayered shielded structure and fifth order filter.

\section{A. Neural Network Performance in the Coupled Line Filter Modeling}

As seen in Figure 7, two neural networks are cascaded to compute the filter dimensions. The first radial basis function neural network has as inputs the quality factor and resonant frequency of the filter. The outputs are the resonator coupling gap $\left(S_{\mathrm{Q}}\right)$, and the resonator length $\left(L_{\mathrm{r}}\right)$. This neural network was trained with 96 points, and it was tested with a different set composed of 84 points. To generate both training and testing sets, the resonator gaps were sampled with 30 equidistant values from 0.055 to $0.40 \mathrm{~mm}$. The line length was sampled with six equidistant values from 5.45 to $5.95 \mathrm{~mm}$. The generation of these sets spent $11 \mathrm{~h}$ and $33 \mathrm{~min}$. The radial basis function neural network was trained with a maximum normalized mean square error of 0.020. In Figure 10, it is verified that the high degree of accuracy is reached by the radial basis function neural network, in the calculation of the coupling gap between the resonator and the input and output ports (gap $S_{\mathrm{Q}}$ ).

We can also see in Figure 11 the precision achieved in the computation of the length resonator when the first neural network is used. We have to remark that the values shown in Figures 10 and 11 correspond to testing points. Therefore, the first radial basis function computes the appropriate physical dimensions when new electrical parameters $\left(Q, \mathrm{f}_{\mathrm{c}}\right)$, different from the training values, are presented as inputs to the neural network.

The second radial basis function neural network computes the coupled lines gaps $\left(S_{\mathrm{K}}\right)$, taking as inputs the resonator length calculated by the first neural network, and the required coupling coefficient. The training and testing sets were generated by means of a sampling process similar to the one used in the first radial basis function neural network. The coupling gap space was sampled from a value of 0.30 to $1.90 \mathrm{~mm}$, with 23 equidistant points. The length space was sampled from 5.45 to $5.95 \mathrm{~mm}$ with six equidistant values. The training set was composed of 72 points, and the testing set was composed of 66 different points. The total time spent to compute the whole training and testing sets was $10 \mathrm{~h}$ and $31 \mathrm{~min}$. The second radial basis function neural network was also trained to reach a normalized mean squared error level of 0.020 . The neural outputs are very close to the direct calculated values, as it can be seen in Figure 12.

Thus, when a certain coupling coefficient is needed, the radial basis function neural network delivers precisely the corresponding resonator coupling gap $\left(S_{\mathrm{K}}\right)$.

\section{B. Neural Network Performance in the Hairpin Filter Modeling}

Again, two neural networks are connected to compute the filter dimensions when hairpin resonators are selected for the design. The first neural network possesses as outputs the tapping distance $(t)$ and the resonator length $\left(L_{\mathrm{r}}\right)$. A total number of 200 points is needed to properly represent the input space of this neural network. The training set was composed of 110 points. The set used to test the generalization capabilities of this neural network is composed of 90 different points. To generate both training and testing sets, the tapping distance was sampled with 20 equidistant values from 4.5 to $10.5 \mathrm{~mm}$. The line resonator length space was sampled with 10 equidistant values from 16.4 to $24.4 \mathrm{~mm}$. The generation of these sets spent $24 \mathrm{~h}$ and $43 \mathrm{~min}$. The maximum normalized mean square error was again set to 0.020 . In Figure 13 , the neural network output corresponding to the tapping distance $(t)$ follows with high precision the testing values, calculated using the techniques explained in "Theory."

In Figure 14, the radial basis function neural network performance in the calculation of the hairpin length resonator is presented. As in the previous case, the neural network attains a high level of accuracy in the computation of the resonator length.

The second radial basis function neural network computes the coupled lines gap $\left(S_{\mathrm{K}}\right)$, in a similar way as the second neural network in the previous exam- 


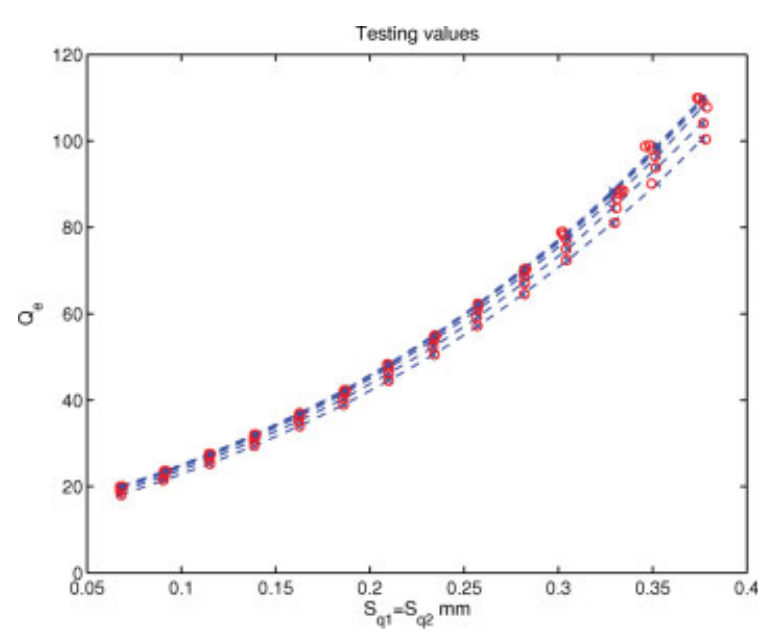

Figure 10. Neural network performance in the testing set of the external quality factor problem when coupled line resonators are used. The structure employed to calculate the relations between the external quality factor and the physical dimensions is depicted in Figure 1(a). Testing values correspond to the dash line with cross points. Neural outputs correspond to circle points. [Color figure can be viewed in the online issue, which is available at www.interscience.wiley.com].

ple. This neural network takes as inputs the resonator length calculated by the first neural network, and the desired coupling coefficient. The input space was sampled to completely represent the variations of the resonator gaps. Furthermore, this sampling process

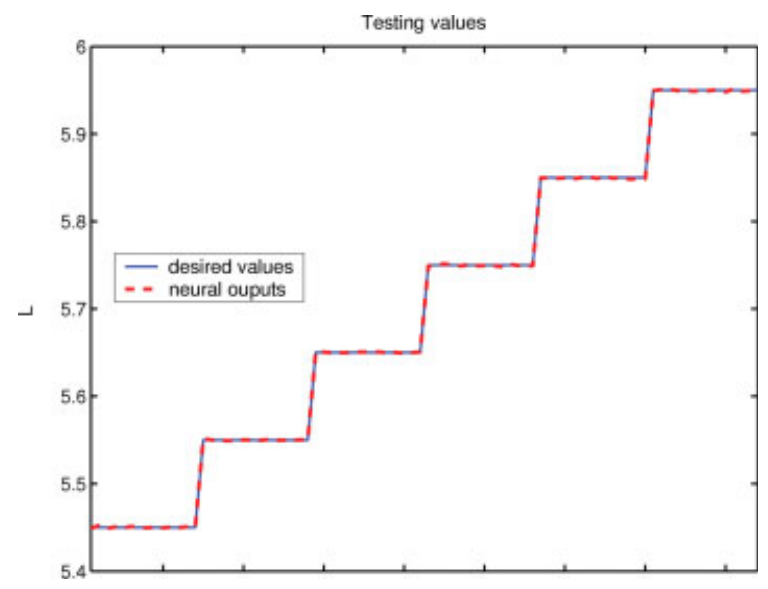

Figure 11. Neural network performance in the testing set corresponding to the calculation of the resonator length $L_{\mathrm{r}}$ when coupled line resonators are used. The structure employed to calculate the relations between the resonator length and the physical dimensions is depicted in Figure 1(a). [Color figure can be viewed in the online issue, which is available at www.interscience.wiley.com].

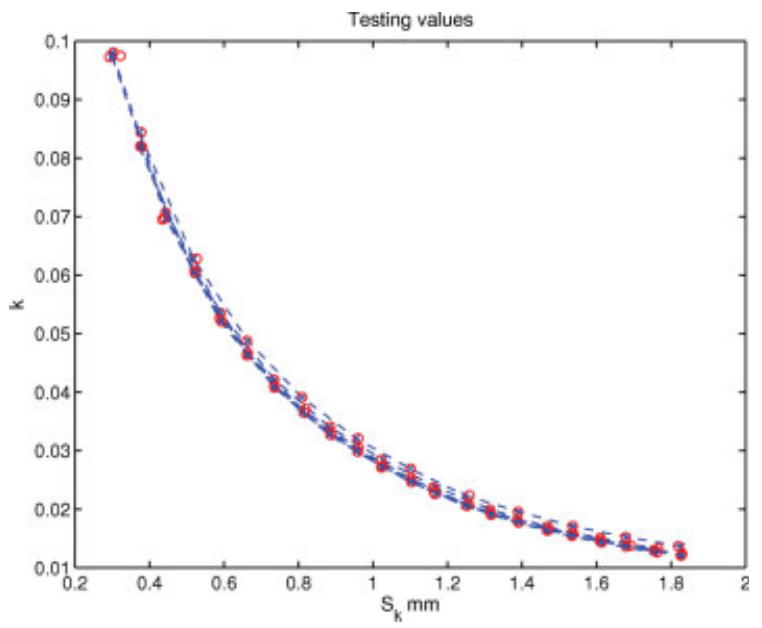

Figure 12. Neural network performance in the testing set of the coupling coefficient problem when coupled line resonators are used. The structure employed to calculate the relations between the resonator separation and the physical dimensions is depicted in Figure 1(b). Testing values correspond to the dash line with cross points. Neural outputs correspond to circle points. [Color figure can be viewed in the online issue, which is available at www.interscience.wiley.com].

generates a testing set to check the generalization ability of the neural network. Thus, the separations space was sampled from a value of 0.20 to $1.00 \mathrm{~mm}$, with 15 equidistant points. The length resonator was sampled from 16.4 to $24.4 \mathrm{~mm}$ with 10 equidistant values. The total time spent to compute the whole training and testing sets was $13 \mathrm{~h}$ and $33 \mathrm{~min}$. A normalized mean squared error level of 0.020 was set as maximum error level. In Figure 15, it is demonstrated the high precision in the calculation of the separation gap, which synthesizes the desired coupling coefficient for a given resonator length.

For both coupled line and hairpin resonators, the radial basis function neural network training time is only few seconds. This is because of the reduced number of training samples needed to represent properly the relations between the electrical parameters and the filter dimensions. The time invested in the generation of the training and testing sets is very large in comparison with the neural training time. But the generation time, as well as the training time, is invested prior to the actual filter design. Therefore, these times do not belong to the real design stage. Once trained, the neural networks calculate the filter dimensions in fractions of a second. If a final optimization procedure is needed, the filter calculated with the neural networks is a very good initial point. This allows reduced designing times as compared with the 


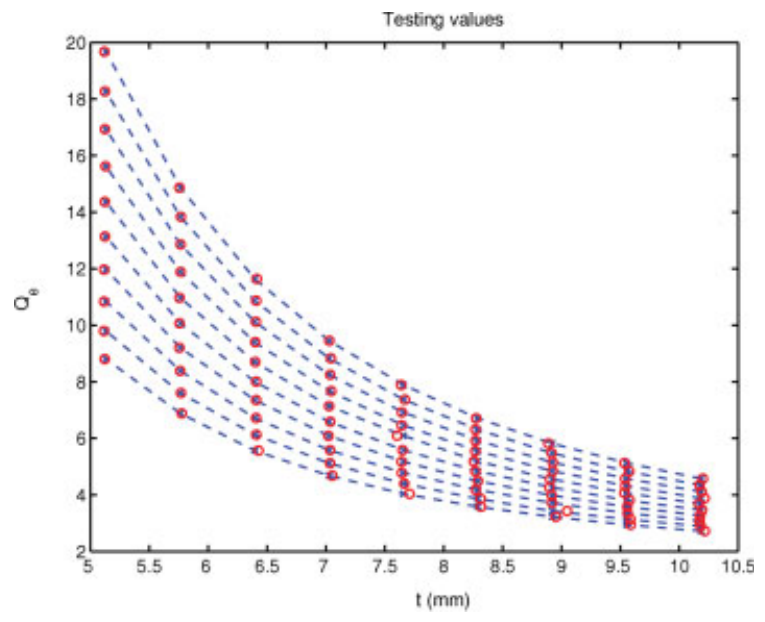

Figure 13. Neural network performance in the testing set of the external quality factor problem when hairpin resonators are used. The structure employed to calculate the relations between the external quality factor and the physical dimensions is depicted in Figure 3(a). Testing values correspond to the dash line with cross points. Neural outputs correspond to circle points. [Color figure can be viewed in the online issue, which is available at www.interscience.wiley.com].

situation when a good initial filter design is not available. In such situations, gradient based optimization techniques might not converge at all to the right sought for solution.

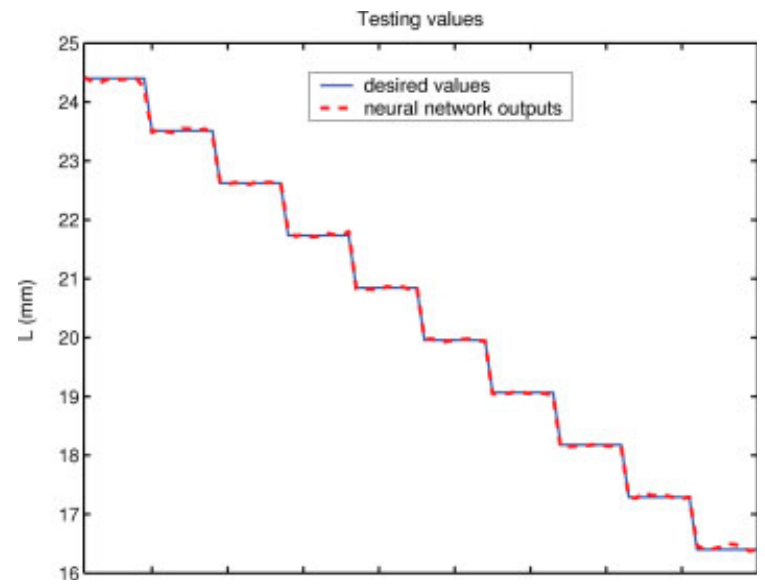

Figure 14. Neural network performance in the testing set corresponding to the calculation of the resonator length $L_{\mathrm{r}}$ when hairpin resonators are used. The structure employed to calculate the relations between the resonator length and the physical dimensions is depicted in Figure 3(a). [Color figure can be viewed in the online issue, which is available at www.interscience.wiley.com].

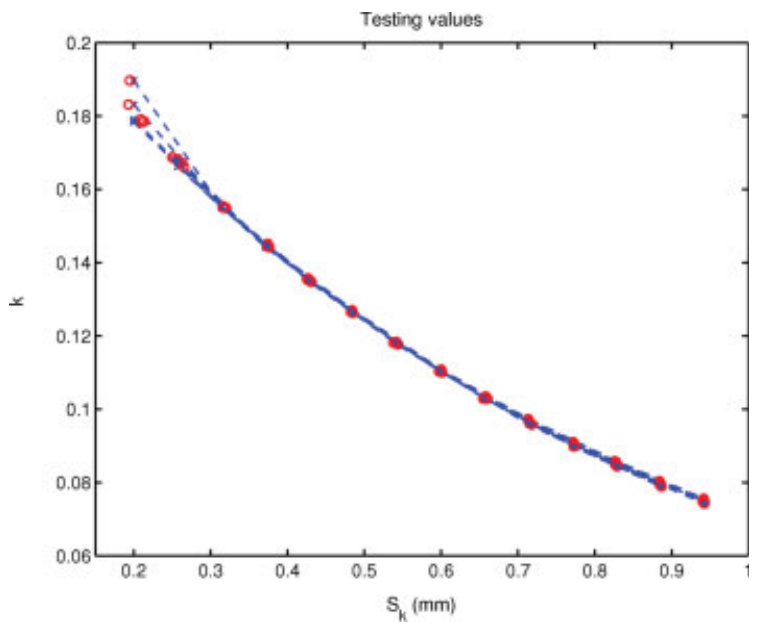

Figure 15. Neural network performance in the testing set of the coupling coefficient problem when hairpin resonators are used. The structure employed to calculate the relations between the resonator separation and the physical dimensions is depicted in Figure 3(b). Testing values correspond to the dash line with cross points. Neural outputs correspond to circle points. [Color figure can be viewed in the online issue, which is available at www.interscience. wiley.com].

\section{FILTER DESIGN}

In the following sections, we present the results obtained for the design of two different filters using the neural networks trained in the previous section.

\section{A. Coupled Line Filter}

To prove the capability of the proposed method for the design of shielded microstrip filters, one four-pole filter was designed in the boxed structure, as sketched in Figure 8. The radial basis function neural network training set allows to design a variety of different transfer function characteristics, with center fre-

TABLE I. Quality Factor, Coupling Coefficients, Separations, and Lengths Corresponding to the Initial Filter and to the Optimized Filter. Fourth Order Filter

\begin{tabular}{lcccc}
\hline & $\begin{array}{c}\text { Initial } \\
\text { Sep. } \\
(\mathrm{mm})\end{array}$ & $\begin{array}{c}\text { Initial } \\
\text { Length } \\
(\mathrm{mm})\end{array}$ & $\begin{array}{c}\text { Optimum } \\
\text { Separation }\end{array}$ & $\begin{array}{c}\text { Optimum } \\
\text { Length }\end{array}$ \\
\hline$Q_{\mathrm{e}}=22.72$ & 0.0992 & 5.4541 & 0.1326 & 5.4650 \\
$k_{12}=0.0405$ & 0.7855 & 5.4541 & 0.7748 & 5.4625 \\
$k_{23}=0.0321$ & 0.9959 & 5.4541 & 0.9941 & 5.4625 \\
$k_{34}=0.0405$ & 0.7855 & 5.4541 & 0.7748 & 5.4650 \\
\hline
\end{tabular}




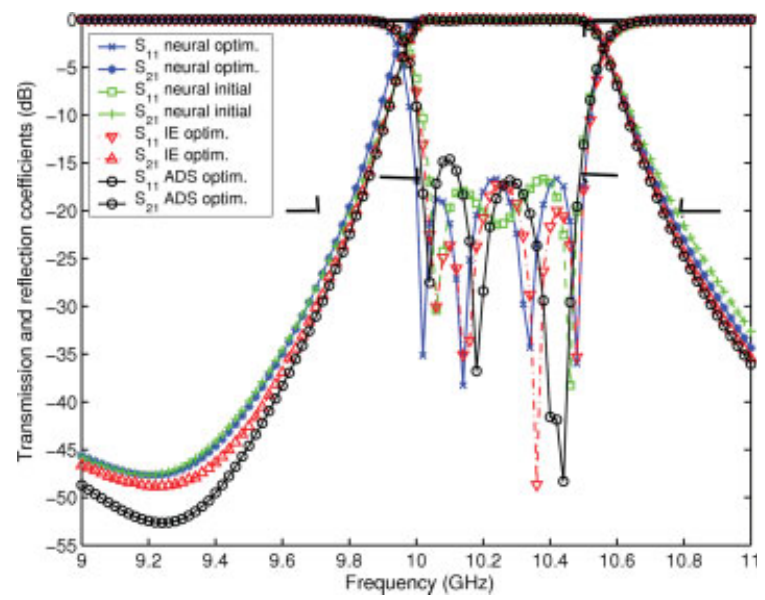

Figure 16. Initial and optimized final solution of the four-pole coupled line filter. Validation is obtained through an integral equation technique and ADS $\odot$ simulation. [Color figure can be viewed in the online issue, which is available at www.interscience.wiley.com].

quency ranging from 9.50 to $10.25 \mathrm{GHz}$. The designed filter has a $5.0 \%$ relative bandwidth centered at $10.25 \mathrm{GHz}$, therefore near the limit of the neural network training set, with a ripple in the pass band of $0.1 \mathrm{~dB}$. The attenuation in the rejection band should be better than $20 \mathrm{~dB}$ in the frequency range of $f<9.70 \mathrm{GHz}$ and $f>10.80 \mathrm{GHz}$. If a Chebyshev response is chosen, the filter must be of fourth order [7]. The resonator lengths and separations calculated with the radial basis function neural network are shown in Table I.

These dimensions correspond to the design of an initial filter, with the response shown in Figure 16 (square and plus sign points). Initially, all the lines possess the same length, because for this type of filters the coupling gap affects little to the resonant frequency of the resonator. The scattering parameters fulfill almost all the specifications. To obtain even better electrical characteristics, an optimization proc-

TABLE II. Quality Factor, Coupling Coefficients, Gap Separations, and Lengths Corresponding to the Initial Filter and to the Optimized Filter. Fifth Order Filter

\begin{tabular}{lcccc}
\hline & $\begin{array}{c}\text { Initial Tap. } \\
\text { and Initial } \\
\text { Parameter }\end{array}$ & $\begin{array}{c}\text { Initial } \\
\text { Length } \\
(\mathrm{mm})\end{array}$ & $\begin{array}{c}\text { Optimum } \\
\text { Tap. } \\
\text { and Sep. }\end{array}$ & $\begin{array}{c}\text { Optimum } \\
\text { Length }\end{array}$ \\
\hline$Q_{\mathrm{e}}=5.755$ & 6.770 & 18.05 & 6.6302 & 18.147 \\
$k_{12}=0.1589$ & 0.2900 & 18.05 & 0.3116 & 18.147 \\
$k_{23}=0.1211$ & 0.5190 & 18.05 & 0.4696 & 18.147 \\
$k_{34}=0.1211$ & 0.5190 & 18.05 & 0.4633 & 18.147 \\
$k_{45}=0.1589$ & 0.2900 & 18.05 & 0.3075 & 18.147 \\
\hline
\end{tabular}

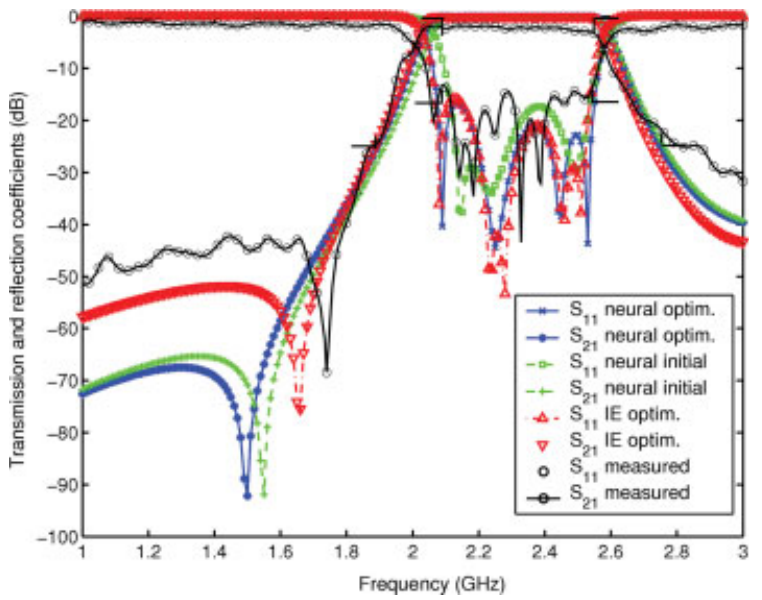

Figure 17. Initial and optimized final solution of the five pole hairpin line filter. Results obtained with an integral equation and measured values are shown for validation. [Color figure can be viewed in the online issue, which is available at www.interscience.wiley.com].

ess is applied to the filter. The optimization step is applied to all the separations and resonator lengths. Thus, different lengths for the resonators are obtained. After only two iterations of the minimax algorithm, as described in Ref. 15, the filter response is improved, as shown also in Figure 16 (cross and asterisk points). Results obtained with an integral equation technique are also shown in Figure 16 (triangle symbols) for validation [16]. To further validate the proposed technique, the final optimized filter response has been simulated with the commercial software Advanced Design System (ADS $($ ), as seen

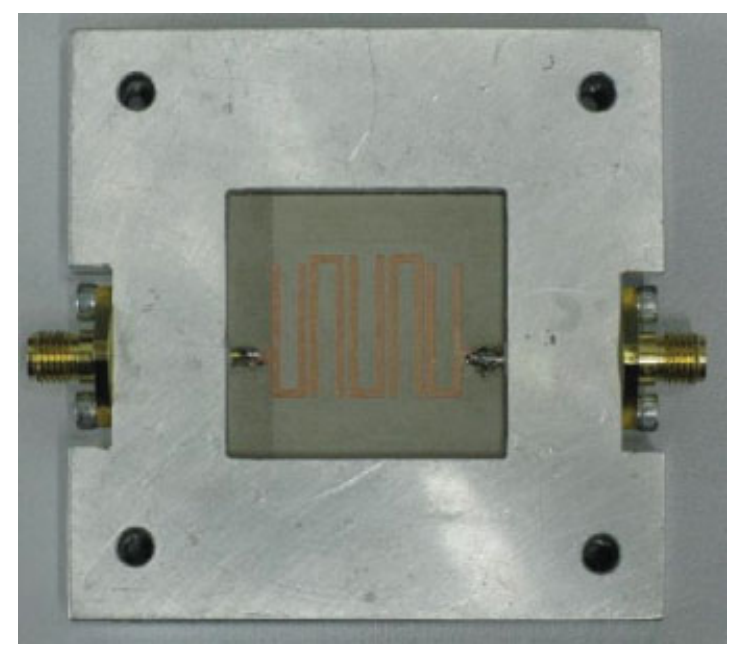

Figure 18. Photo of the manufactured five-pole hairpin filter. [Color figure can be viewed in the online issue, which is available at www.interscience.wiley.com]. 
in Figure 16 (circle symbols). We can observe good agreement between all responses.

It is important to point out that, although the initial filter does not fulfill completely the specifications, it is a very good initial point for an optimization step. The differences between the initial values and the final optimized dimensions are very small (less than $2.0 \%$ ). In this manner, fast gradient based techniques can be applied with excellent results.

\section{B. Hairpin Filter}

Using the neural networks shown in Figure 7, one hairpin filter was designed in the boxed structure sketched in Figure 9. The radial basis function neural networks were trained to calculate the dimensions of filters with different transfer functions and central frequency ranging from 1.60 to $2.55 \mathrm{GHz}$. The hairpin filter has a $20.0 \%$ relative bandwidth centered at $2.30 \mathrm{GHz}$. The attenuation in the rejection band should be better than $25 \mathrm{~dB}$ in the frequency range of $f<1.90 \mathrm{GHz}$ and $f>2.75 \mathrm{GHz}$. As in the coupled line filters, a Chebyshev transfer function is chosen. The order of the filter must be five to attain the desired attenuation in both transmission and rejection bands [7]. In Table II, the dimensions computed by the neural networks for the tapping length, resonator lengths, and resonator separations are shown. All the resonators have the same initial length, because again, the coupling gaps hardly affect the resonant frequency of the resonators.

The magnitude of the transmission and reflection coefficients for the initial filter are shown in Figure 17. After nine iterations of the minimax optimization, the hairpin filter fulfills all the specifications for both rejection and transmission bands (see Fig. 17). In this case, to maintain a limited number of optimization variables, all the resonators are forced to possess the same length. Small differences are observed between the initial and final optimized physical dimensions. The optimization procedure must correct the response corresponding to the initial filter, only very slightly. Thus, although a final optimization step is needed, the final response is obtained in a very robust way. This clearly shows the capabilities of the neural method, for practical designs.

To verify the developed technique, the five pole hairpin filter was manufactured as seen in Figure 18. The results included in Figure 17 show good agreement between the measured values (circle points) and the simulated responses. The fabricated prototype exhibits a minimum measured insertion loss within the passband of $-1.79 \mathrm{~dB}$.

\section{CONCLUSIONS}

In this work, a new neural network technique for the design of shielded bandpass filters is proposed. Neural networks are used to represent the relations between external quality factors and coupling coefficients, with the physical dimensions of all the resonators of the filter. Once the neural networks have been trained for a given structure and for a fixed type of resonators, different filtering transfer functions can be designed in a fast and accurate way. Another neural network method is employed to analyze the filters and to provide the training and testing sets, accelerating the whole design process. The design of one coupled line bandpass filter and one hairpin bandpass filter, in two different shielded structures, demonstrate the validity and usefulness of the new neural network technique. The developed technique can be extended to any other kind of filter based coupled resonators.

\section{REFERENCES}

1. M.M. Vai, W.S. Wu, L. Bin, and S. Prasad, Reverse modeling of microwave circuits with bidirectional neural network models, IEEE Trans Microwave Theory Tech 46 (1998), 1492-1494.

2. S. Selleri, S. Manetti, and G. Pelosi, Neural network applications in microwave device design, Int $\mathrm{J}$ RF Microwave CAE 12 (2002), 90-97.

3. G. Fedi, A. Gaggelli, S. Manetti, and G. Pelosi, Direct-coupled cavity filters design using a hybrid feedforward neural network finite elements procedure, Int J RF Microwave CAE 9 (1999), 287-296.

4. Y. Wang, M. Yu, H. Kabir, and Q.-J. Zhang, Effective design of cross-coupled filter using neural networks and coupling matrix, IEEE Int Microwave Symp 1-5 (2006), 1431-1434.

5. H. Kabir, Y. Wang, M. Yu, and Q.-J. Zhang, Neural network inverse modeling and applications to microwave filter design, IEEE Trans Microwave Theory Tech 56 (2008), 867-879.

6. P.M. Watson, C. Cho, and K.C. Gupta, Electromagnetic-artificial neural network model for synthesis of physical dimensions for multilayer asymmetric coupled transmission structures, Int J RF Microwave CAE 9 (1999), 175-186.

7. G.L. Matthaei, L. Young, and E.M.T. Jones, Microwave filters, impedance-matching networks, and coupling structures, Artech House, Norwood, MA, 1980.

8. J.S. Hong and M.J. Lancaster, Microstrip filters for rf/ microwave applications, Wiley, New York, 2001.

9. T.M. Weller, Edge-coupled coplanar waveguide bandpass filter design, IEEE Trans Microwave Theory Tech 48 (2000), 2453-2458. 
10. J.-T. Kuo and H.-S. Cheng, Design of quasi-elliptic function filters with a dual-passband response, IEEE Microwave Wireless Compon Lett 14 (2004), 472-474.

11. J.P. Garcia, F.D.Q. Pereira, D.C. Rebenaque, J.L.G. Tornero, and A.A. Melcon, A neural-network method for the analysis of multilayered shielded microwave circuits, IEEE Trans Microwave Theory Tech 54 (2006), 309-320.

12. J. Bandler, R. Biernacki, S.H. Chen, J.D.G. Swanson, and S. Ye, Microstrip filter design using direct em field simulation, IEEE Trans Microwave Theory Tech 42 (1994), 1353-1359.

13. J. Ureel and D.D. Zutter, Gradient-based minimax optimization of planar microstrip structures with the use of electromagnetic simulations, Int J RF Microwave CAE 7 (1997), 29-36.

14. J.T. Alos and M. Guglielmi, Simple and effective embased optimization procedure for microwave filters, IEEE Trans Microwave Theory Tech 45 (1997), 856858.

15. J.W. Bandler, W. Kellerman, and K. Madsen, A superlinearly convergent minimax algorithm for microwave circuit design, IEEE Trans Microwave Theory Tech 33 (1985), 1519-1530.

16. A.A. Melcon, J.R. Mosig, and M. Guglielmi, Efficient cad of boxed microwave circuits based on arbitrary rectangular elements, IEEE Trans Microwave Theory Tech 47 (1999), 1045-1058.

\section{BIOGRAPHIES}

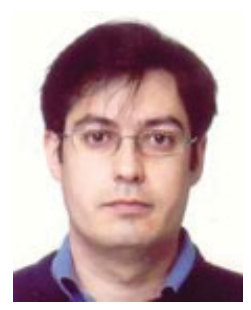

Juan Pascual García was born in Castellón, Spain, in 1975. He received the Telecommunications Engineer degree from the Technical University of Valencia (UPV), Valencia, Spain, in 2001. He is currently working toward the Ph.D. degree at the Technical University of Cartagena (UPCT), Cartagena, Spain. In 2003, he joined the Communications and Information Technologies Department, UPCT, as a Research Assitant and then as an Assistant Professor. His research interests include neural networks, genetic algorithms, and their applications in the analysis and development of computeraided design tools for microwave circuits and antennas.

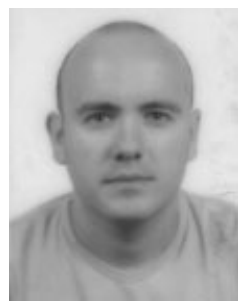

Fenando D. Quesada Pereira was born in Murcia, Spain, in 1974. He received the Telecommunications Engineer degree from the Technical University of Valencia (UPV), Valencia, Spain, in 2000, and the Ph.D. degree from the Technical University of Cartagena (UPCT), Cartagena, Spain in 2007. In 1999, he joined the Radiocommunications Department, UPV, as a Research Assistant, where he was involved in the development of numerical methods for the analysis of anechoic chambers and tag antennas. In 2001, he joined the Communications and Information Technologies Department, UPCT, initially as a Research Assistant, and then as an Assistant Professor. In 2005, he spent six months as a Visiting Scientist with the University of Pavia, Pavia, Italy. His current scientific interests include integral equation numerical methods for the analysis of antennas and microwave devices.

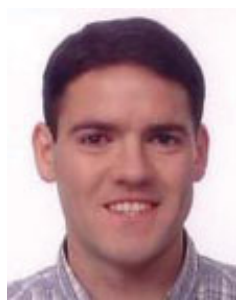

David Cañete Rebenaque was born in Valencia, Spain, in 1976. He received the Telecommunications Engineer degree from the Technical University of Valencia (UPV), Valencia, Spain, in 2000 and is currently working toward the Ph.D. degree at the Technical University of Cartagena (UPCT), Cartagena, Spain. During 2001, he was an RF Engineer with a mobile communications company. In 2002, he joined the Communications and Information Technologies Department, UPCT, initially as a Research Assistant, and then as an Assistant Professor. His research interests include the analysis and design of microwave circuits and active antennas.

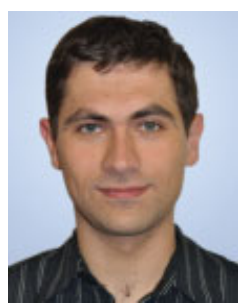

Juan Sebastián Gómez Díaz was born in Ontur (Albacete), Spain, in 1983. He received the Telecommunications Engineer degree (with honors) from the Technical University of Cartagena (UPCT), Spain, in 2006, where he is currently working toward the Ph.D. degree. In 2007 he joined the Telecommunication and Electromagnetic group (GEAT), UPCT, as a Research Assistant. From November 2007 to October 2008, he was at Poly-Grames, École Polytechnique de Montréal as a visiting Ph.D. student, where he was involved in the impulseregime analysis of linear and nonlinear metamaterial-based devices and antennas. His current scientific interests also include Integral Equation and Numerical Methods and their application to the analysis and design of microwave circuits and antennas.

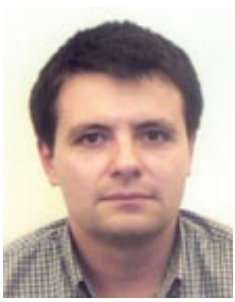

Alejandro Álvarez Melcón received the Telecommunications Engineer degree from the Technical University of Madrid (UPM), Spain, in 1991, and the Ph.D. degree in electrical engineering from the Swiss Federal Institute of Technology, Lausanne, Switzerland, in 1998. From 1991 to 1993 , he was with the Radio Frequency Systems Division, European Space Agency (ESA/ESTEC), Noordwijk. From 1993 to 1995, he was with the Space Division, Industry Alcatel Espacio, Madrid, and was also with the ESA. From 1995 to 1999, he was with the Swiss Federal Institute of Technology, Ecole Polytechnique Fédérale de Lausanne (EPFL), Switzerland. In 2000, he joined the Technical University of Cartagena, Spain, where he is currently developing his teaching and research activities. Dr. Melcon was the recipient of the Best Paper Award for the best contribution to the JINA'98 International Symposium on Antennas, and the Colegio Oficial de Ingenieros de Telecomunicacion (COIT/AEIT) Award to the best Ph.D. thesis. 